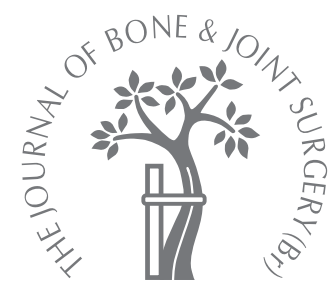

\title{
The effect of the offset humeral head on the micromovement of pegged glenoid
} components

\author{
A COMPARATIVE STUDY USING RADIOSTEREOMETRIC ANALYSIS
}

D. Nuttall, J. F. Haines, I. A. Trail

From Hand and

Upper Limb

Research Unit,

Wrightington

Hospital, Wigan, England
D. Nuttall, PhD, Research Fellow

J. F. Haines, FRCS, Consultant Orthopaedic Surgeon I. A. Trail, FRCS, Consultant Orthopaedic Surgeon Hand and Upper Limb Research Unit

Wrightington Hospital, Hall Lane, Appley Bridge, Wigan WN6 9EP, UK.

Correspondence should be sent to Dr D. Nuttall; e-mail: uplimb_dn@hotmail.com

(C)2009 British Editorial Society of Bone and Joint Surgery doi:10.1302/0301-620X.91B6. $22060 \$ 2.00$

$J$ Bone Joint Surg $[\mathrm{Br}]$ 2009;91-B:757-61. Received 14 November 2008; Accepted after revision 10 March 2009

In a prospective study between 2000 and 2005, 22 patients with primary osteoarthritis of the shoulder had a total shoulder arthroplasty with a standard five-pegged glenoid component, 12 with non-offset humeral head and ten with offset humeral head components. Over a period of 24 months the relative movement of the glenoid component with respect to the scapula was measured using radiostereometric analysis.

Nine glenoids needed reaming for erosion. There was a significant increase in rotation about all three axes with time $(p<0.001)$, the largest occurring about the longitudinal axis (anteversion-retroversion), with mean values of $3.8^{\circ}$ and $1.9^{\circ}$ for the non-offset and offset humeral head eroded subgroups, respectively. There was also a significant difference in rotation about the anteversion-retroversion axis $(p=0.01)$ and the varus-valgus $(p<0.001)$ $z$-axis between the two groups. The offset humeral head group reached a plateau at early follow-up with rotation about the z-axis, whereas the mean of the non-offset humeral head group at $\mathbf{2 4}$ months was three times greater than that of the offset group accounting for the highly significant difference between them.

Replacement of the shoulder is firmly established as a reliable method of treatment for a variety of conditions affecting the joint. ${ }^{1-8}$ Loosening of the glenoid component remains a considerable concern since radiolucent lines are often seen on the initial post-operative films and at follow-up. ${ }^{9,10}$ Little is known about the mechanism of this loosening. One large series described significant differences in glenoid lucency around keeled and pegged components. ${ }^{5}$ Other factors which may also be important are the size and position of the replacement of the humeral head.

Early prosthetic systems used a single radius of curvature for the humeral head with a matching radius of curvature of the glenoid. ${ }^{11}$ That of the humeral head and the length of the humeral neck determine the dimensions of the articular surface of the head. Although these parameters vary widely among individuals, the ratio of the diameter of the head to the length of the neck is fairly constant. ${ }^{12}$ In anatomical studies, the humerus has been shown to have a combined offset to the longitudinal axis of the humerus both posteriorly and medially ${ }^{13}$ and, as a consequence, modular humeral components which are adaptable to the individual anatomy have been developed. These attempt to restore the original three-dimensional geometry of the proximal humerus. The effect of these offsets on the longevity of the glenoid component and on the clinical outcome is not known.

Our study was designed to determine the effects of offset of the humeral head on the micromovement of standard five-pegged glenoid components by using radiostereometric analysis (RSA) ${ }^{14}$ to measure the position of rigid bodies in three dimensions and to compare this early movement with that of a series of comparable implants in which a nonoffset humeral head had been used. A secondary aim was to describe the early movement of the glenoid components using exploratory principal-component analysis ${ }^{15}$ which can be used to indicate implants at risk.

\section{Patients and Methods}

In a prospective study between 2000 and 2005, 30 patients with primary osteoarthritis who had a total shoulder arthroplasty were studied by RSA. A total of 15 patients had a non-offset humeral head before $2003^{14}$ and 15 an offset humeral head after that date. Approval of the ethical committee had been obtained and the patients gave informed consent. Good glenoid bone stock, sufficient to introduce a glenoid component, and an intact and functioning rotator cuff were required. A total of eight patients was excluded from the study including 


\begin{tabular}{|c|c|c|c|c|c|c|}
\hline \multirow[b]{2}{*}{$\begin{array}{l}\text { Diameter of the } \\
\text { humeral head }(\mathrm{mm})\end{array}$} & \multicolumn{3}{|c|}{$\begin{array}{l}\text { NOHH } \\
\text { depth }\end{array}$} & \multicolumn{3}{|c|}{$\begin{array}{l}\text { OHH } \\
\text { depth }\end{array}$} \\
\hline & $\mathbf{S}$ & $\mathbf{M}$ & $\mathbf{L}$ & 16 & 18 & 21 \\
\hline 40 & 1 & 3 & 0 & 0 & 1 & 0 \\
\hline 44 & 0 & 2 & 1 & 1 & 2 & 1 \\
\hline 48 & 0 & 3 & 0 & 0 & 3 & 1 \\
\hline 52 & 0 & 0 & 1 & 0 & 0 & 1 \\
\hline 56 & 0 & 0 & 1 & 0 & 0 & 0 \\
\hline
\end{tabular}

three who died, two who withdrew because of failing health before the end of the study, two who had unstable placement of the marker beads and one in whom the glenoid component could not be clearly visualised. This left 22 patients in the study. The non-offset humeral head group had a male-to-female ratio of 4:8 with a mean age of 69 years (58 to 84). In the offset humeral head group the male-to-female ratio was $3: 7$ and the mean age was 65 years (60 to 80$)$.

Operative technique. The operations were undertaken by two of the authors (IAT, JFH) in a standardised fashion. The patients were placed in the beach-chair position and a deltopectoral approach was used. None had deltoid release, although most had a partial release of pectoralis major. The biceps tendon was present in all but one patient and the rotator cuff in all. Supraspinatus was found to be thinned but intact in two patients. An anterior and inferior capsular release was carried out. Preparation of the glenoid and humerus was undertaken as described in the manufacturer's guidelines. Any residual soft-tissue remnants such as labral remnants were removed from the glenoid. A central hole was then defined and using the appropriately sized reamer any remaining cartilage was removed. Any erosion was corrected by tilting the reamer appropriately. The glenoid was then drilled to allow insertion of the five-pegged component which was cemented into place. The Global shoulder arthroplasty system (DePuy International, Leeds, United Kingdom) was used in all cases. The humeral components were inserted uncemented in $30^{\circ}$ of retroversion. The size of the humeral head was correlated with the dimensions of the excised head allowing appropriate soft-tissue balance. In the first group the heads were not offset and in the second they were generally offset posterosuperiorly. The range of head size for each group is shown in Table I. With the Global system there is a difference of $6^{\circ}$ in the diameter of curvature between the humeral and glenoid components. Post-operatively, all patients began a passive and supervised early active mobilisation programme.

The glenoid bone stock was assessed by either axial plain radiographs or a CT scan, as well as during the operation. The rotator cuff was assessed both clinically and during surgery both visually and by palpation. At the time of operation, six glenoids in the non-offset group and three in the offset humeral head group were eroded and required corrective reaming. Using the classification of Walch et $\mathrm{al},{ }^{16}$ the erosions in the former group were classified as anterior in three and type B1 in three. In the offset humeral head group there were two anterior erosions and one type B1. Clinical evaluation included assessment of pain on a visual analogue scale, movement in all planes, strength and function. This allowed calculation of the Constant-Murley ${ }^{17}$ and American Shoulder and Elbow Surgeons (ASES) scores. ${ }^{18}$ Assessments were undertaken pre-operatively and at the time of the radiological review at follow-up.

RSA technique. At operation, tantalum beads $(1 \mathrm{~mm})$ were inserted into the scapula, typically with two in the acromion, two in the glenoid and one in the coracoid. The beads were inserted using a drill and a special delivery system. Beads were also inserted into the pegs of the implant. ${ }^{14}$ Stereo pairs of radiographs were obtained using the uniplanar UmRSA Table 43 (RSA Biomedical, Umea, Sweden). RSA radiographs were taken post-operatively and after three, six, 12 and 24 months. They were digitised and analysed using the UmRSA digital version 5 system (RSA Biomedical). The relative movement of the glenoid component with respect to the scapula was measured. The UmRSA system predefines linear movement as translation along the following axes: transverse ( $\mathrm{x}$-axis, mediolateral translation), longitudinal (y-axis, proximodistal translation) and sagittal (z-axis, anteroposterior translation). Similarly, rotation is defined about the three axes: transverse ( $\mathrm{x}$-axis, anteroposterior), longitudinal (y-axis, anteversion-retroversion) and sagittal (z-axis, varus-valgus). The technique of RSA of the glenoid has been described previously ${ }^{14}$ and has been shown to be reproducible and accurate with the longitudinal axis having the largest critical level $(p<0.05)$ for translation equal to $0.13 \mathrm{~mm}$ and rotation equal to $1.2^{\circ}$. Statistical analysis. Clinical outcome measures were examined by use of a paired $t$-test on pre- and post-operative measures. Absolute RSA measurements were analysed using parametric methods. ${ }^{14,19}$ Differences in the early rates of loosening for translation and rotation of the centroid between implants were determined by using analysis of variance (ANOVA).The within-subject factor was followup at three, six, 12 and 24 months, and two betweensubject factors were the offset or non-offset humeral head 
Table II. Mean values of movement and shoulder scores before and after operation for the two groups. Pain was measured using a $10 \mathrm{~cm}$ visual analogue scale. Movement was measured in degrees except for internal rotation, which was measured on the Constant-Murley ten-point scale. The American shoulder and elbow surgeons (ASES) and Constant-Murley scores ranged from 0 to 100

\begin{tabular}{|c|c|c|c|c|}
\hline \multirow[b]{2}{*}{ Diameter of the humeral head (mm) } & \multicolumn{2}{|l|}{ NOHH $^{*}$} & \multicolumn{2}{|l|}{$\mathrm{OHH}^{\dagger}$} \\
\hline & Pre-operative & Post-operative & Pre-operative & Post-operative \\
\hline Pain & 5.8 & 0.8 & 7.4 & 0.8 \\
\hline Abduction & 65 & 103 & 57 & 102 \\
\hline Flexion & 78 & 116 & 72 & 117 \\
\hline ASES score & 33 & 88 & 26 & 82 \\
\hline Constant-Murley score & 29 & 61 & 23 & 71 \\
\hline
\end{tabular}

* NOHH, non-offset humeral head

$\mathrm{\dagger} \mathrm{OHH}$, offset-humeral head

Table III. Mean values $\left({ }^{\circ}\right)$ of rotation of rigid body in three dimensions for the standard five-pegged glenoid component and analysis of variance comparing micromovement with time for the two groups, according to the treatment for glenoid erosion at the time of operation

\begin{tabular}{|c|c|c|c|c|c|c|c|c|c|c|}
\hline & \multirow[b]{2}{*}{ Component $^{*}$} & \multirow[b]{2}{*}{ Erosion } & \multirow[b]{2}{*}{ Number } & \multicolumn{4}{|c|}{ Time (mths) } & \multicolumn{3}{|l|}{ p-value } \\
\hline & & & & 3 & 6 & 12 & 24 & Within time & Between component & Between erosion \\
\hline \multicolumn{11}{|l|}{ Mean rotation } \\
\hline \multirow{3}{*}{ Anterior/posterior $(x)$} & $\mathrm{NOHH}$ & Yes & 4 & 0.2 & 1.0 & 1.0 & 1.0 & & & \\
\hline & $\mathrm{OHH}$ & No & 7 & 0.7 & 1.0 & 0.6 & 0.7 & & & \\
\hline & $\mathrm{OHH}$ & Yes & 3 & 0.2 & 0.4 & 0.6 & 0.2 & $<0.001$ & 0.19 & 0.03 \\
\hline \multirow[t]{4}{*}{ Ante/retroversion (y) } & $\mathrm{NOHH}$ & No & 6 & 1.1 & 0.9 & 1.0 & 1.7 & & & \\
\hline & $\mathrm{NOHH}$ & Yes & 4 & 1.1 & 2.6 & 2.6 & 3.8 & & & \\
\hline & $\mathrm{OHH}$ & No & 7 & 0.9 & 0.7 & 1.8 & 1.9 & & & \\
\hline & $\mathrm{OHH}$ & Yes & 3 & 0.4 & 0.4 & 0.9 & 0.8 & $<0.001$ & 0.01 & 0.30 \\
\hline \multirow[t]{3}{*}{ Varus/valgus (z) } & $\mathrm{NOHH}$ & No & 6 & 0.7 & 1.3 & 1.6 & 1.8 & & & \\
\hline & $\mathrm{NOHH}$ & Yes & 4 & 0.5 & 1.1 & 1.8 & 2.6 & & & \\
\hline & $\mathrm{OHH}$ & Yes & 3 & 0.4 & 0.7 & 0.9 & 0.8 & $<0.001$ & $<0.001$ & 0.96 \\
\hline
\end{tabular}

* $\mathrm{NOHH}$, non-offset humeral head; $\mathrm{OHH}$, offset humeral head

groups and the presence or absence of glenoid erosion. RSA studies can report absolute or signeddata ${ }^{19,20}$ which do not have to be mutually exclusive. Signed RSA measurements ${ }^{20}$ were analysed using principal-component analysis of the six parameters of movement of the glenoid segment. This multivariate technique has previously been applied to the glenoid and identifies linear associations between the six degrees of freedom of centroid movement and produces summary components which are orthogonal to each other. ${ }^{14}$ The principal-component analysis produces coordinates for the whole dataset and these can provide a clear indication of the implants at risk as well as describing the complex movements occurring over time.

\section{Results}

The clinical outcome for all patients is shown in Table II. With a minimum follow-up of two years the pain scores, the range of movement, the Constant-Murley score, and the selfassessed ASES score all improved significantly (paired $t$-test, $\mathrm{p}<0.001$ ) for both groups of patients. There was no signifi- cant difference in clinical outcome between the two groups. The offset group had a mean Constant-Murley score which was ten points greater than that of the non-offset group.

The ANOVA results of the glenoid rigid-body rotations are shown in Table III. There was a significant increase in rotation about all three axes over time. The largest rotations occurred about the longitudinal axis (anteversionretroversion) with mean values of $3.8^{\circ}$ and $1.9^{\circ}$ for the nonoffset and offset humeral head eroded subgroups, respectively. There was also a significant difference in rotation between the former and latter groups about the anteversion-retroversion axis $(p=0.01)$ and most notably about the varus-valgus $(\mathrm{p}<0.001)$ axis. The mean rotation about the $\mathrm{z}$-axis is shown in Figure 1 in which the offset humeral head group reached a plateau early in follow-up and showed a small $95 \%$ confidence interval (CI). The mean of the non-offset humeral head group at 24 months was three times greater than that of the offset group accounting for the highly significant difference between the groups as shown in Table III. 


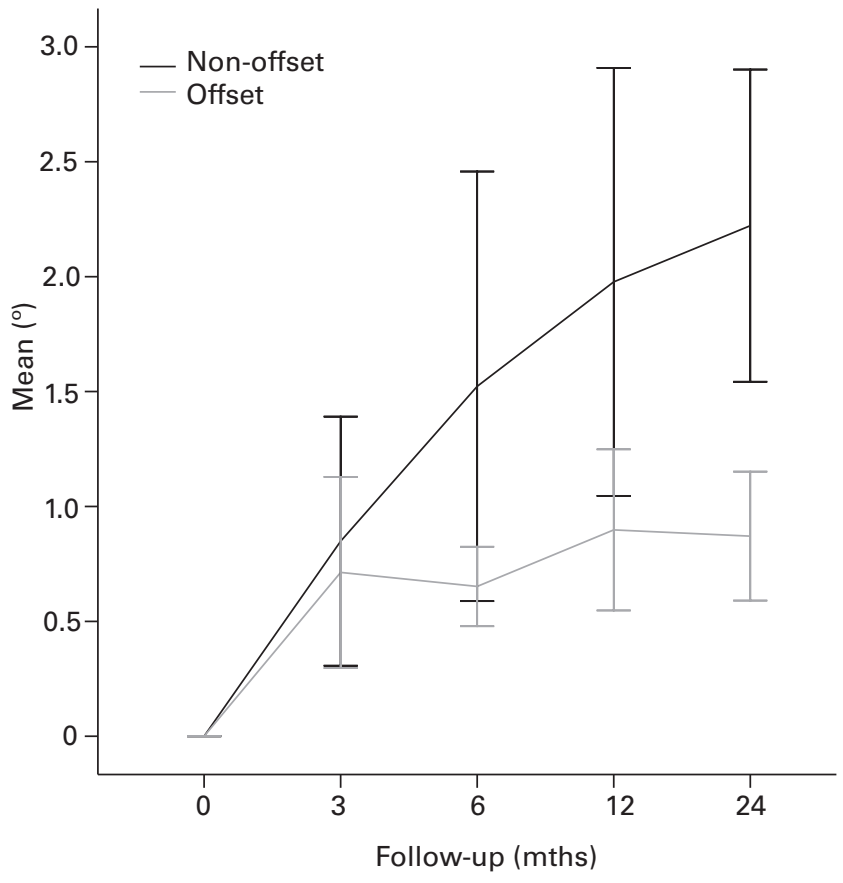

Fig. 1

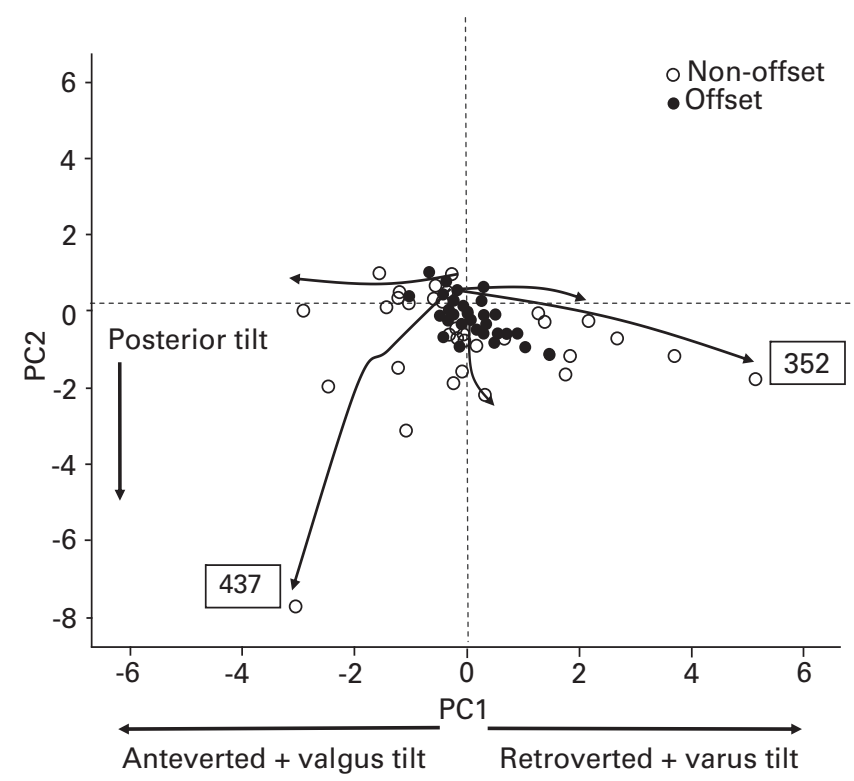

Fig. 2

Principal-component (PC) co-ordinate graph showing the full radiostereometric analysis dataset.
Graph showing the mean rotation about the $z$-axis (varus/valgus tilt) over time for the two groups. The error bars show the $95 \%$ confidence intervals.

Most glenoid components from both groups initially moved and then reached a plateau by 24 months. There were, however, two notable exceptions, both in the non-offset group. In one patient a glenoid component had perforated the cortical bone at the time of surgery and in another who had resumed heavy lifting, superior subluxation developed.

Principal-component analysis was performed on all of the dataset. The first component accounted for $43 \%$ of all variations and was dominated by three linear interactions, namely anteversion/retroversion about the $y$-axis together with varus rotation and translation about the $\mathrm{z}$-axis. The second component, unrelated to the first, accounted for $19 \%$ of all residual variation and was composed of a linear interaction between anterior/posterior tilt about the $\mathrm{x}$-axis and translation along the y-axis. The principal component co-ordinate plot of the whole dataset obtained from this analysis is shown in Figure 2.

The principal co-ordinate score has zero mean and unit variance and can be considered to be similar to a $\mathrm{z}$-score with outliers indicated when the score is greater than two. Five nonoffset humeral head components had a score greater than two, but no offset components. The two non-offset components which had continued to move had scores greater than five which suggested that these implants were at risk.

\section{Discussion}

Aseptic loosening of a glenoid component is a common complication of total shoulder replacement in the medium to long term. ${ }^{9,21-23}$ While there is a disparity between the incidence of radiolucent lines around glenoid components and the rate of revision, there is increasing evidence that these lines become more pronounced with time. ${ }^{2,24-26}$ Overall, it appears that loosening of the glenoid is inevitable and will, if the patient survives, lead to the onset of further symptoms. The factors affecting loosening of the glenoid are numerous ${ }^{27}$ and include the design of the component, surgical technique of preparation of the glenoid and cementing, the shape and position of the humeral component and other factors. Both clinical and RSA studies have previously shown the advantage of the pegged over the keeled component. ${ }^{9,14,28}$

Replacement of the head of the humerus in an anatomical position may have an effect on the glenoid component. Our study compared two groups of patients one of which had a non-anatomical replacement of the humeral head and the other an anatomical replacement using an offset head. The smallest movement occurred about the $\mathrm{x}$-axis in both groups, the pegged design controlling rotation about this axis. Our results clearly showed less micromovement with a more anatomical humeral head about the $\mathrm{z}$-axis with a highly significant difference between the two groups; this was not observed about the y-axis. The reason for this remains unclear although it may be that the offset head, while improving the anatomy of the replacement does not address tilt. Improvements in the design of the humeral head now allow correction for version, offset and tilt.

Our study also showed that the use of a more anatomical head did not appear to confer an improved range of movement. This was a surprising finding although our samples 
are small. The ten-point difference in Constant score was not statistically significant, however, the power of the $t$-test was only 0.3 indicating a type- 2 error. Each group would require to have 33 patients to obtain a power of 0.8 .

The correction of glenoid erosion appears to have a detrimental effect on glenoid movement. Glenoids which are inserted after a surgical correction for erosion seem to migrate more than those which do not need this, as has been noted previously. ${ }^{4,14}$ The reason remains unclear. It would seem logical that correct realignment of the glenoid component would improve its survivorship. One explanation of our finding may be that this correction was done manually by the naked eye. While there was thought to be some improvement, this could not be quantified. In order to assess this the patient would need to undergo CT to determine the glenoid version before and after insertion of the prosthesis using the techniques described by Nyffeler et al. ${ }^{29}$

We have used principal-component analysis which makes maximum use of signed data in describing summary patterns of micromovement for all six degrees of freedom of movement. The principal component co-ordinates of the dataset were used to produce an at-risk index in chart form as shown in Figure 2. The pattern of migration of each glenoid can easily be seen at a glance using this method. The wandering arrows in Figure 2 describe the migration patterns of five non-offset humeral head glenoid components, two of which seem to be at risk and are labelled in the figure as operations 352 and 437.

With further improvements in the design of the glenoid component, better preparation and cementing, with management of the humeral head and surrounding soft tissues, the survivorship of glenoid components in total shoulder replacement will continue to improve. Further designs of the glenoid component should fully address the pleiomorphic nature of the glenoid notch, ${ }^{12,30}$ specifically its pear-drop rather than circular shape.

No benefits in any form have been received or will be received from a commercial party related directly or indirectly to the subject of this article.

\section{References}

1. Barrett WP, Franklin JL, Jackins SE, Wyss CR, Matsen FA 3rd. Total shoulder arthroplasty. J Bone Joint Surg [Am] 1987;69-A:865-72.

2. Cofield R. Total shoulder arthroplasty with the Neer prosthesis. J Bone Joint Surg [Am] 1984;66-A:899-906.

3. Neer CS 2nd. Unconstrained shoulder arthroplasty. Instr Course Lect 1985;34:278-86.

4. Haines JF, Trail IA, Nuttall D, Birch A, Barrow A. The results of arthroplasty in osteoarthritis of the shoulder. J Bone Joint Surg [Br] 2006;88-B:496-501.

5. Trail IA, Nuttall D. The results of shoulder arthroplasty in patients with rheumatoid arthritis. J Bone Joint Surg [Br] 2002;84-B:1121-5.

6. Boileau P, Sinnerton RJ, Chuinard C, Walch G. Arthroplasty of the shoulder. J Bone Joint Surg [Br] 2006;88-B:562-75.
7. Raiss $\mathbf{P}$, Aldinger PR, Kasten P, Rickert $\mathbf{M}$, Loew $\mathbf{M}$. Total shoulder replacement in young and middle-aged patients with glenohumeral osteoarthritis. J Bone Joint Surg [Br] 2008;90-B:764-9.

8. Mullett H, Levy O, Raj D, et al. Copeland surface replacement of the shoulder: results of an hydroxyapatite-coated cementless implant in patients over 80 years of age. J Bone Joint Surg [Br] 2007;89-B:1466-9.

9. Lazarus MD, Jensen KL, Southworth C, Matsen FA 3rd. The radiographic evaluation of keeled and pegged glenoid component insertion. J Bone Joint Surg [Am] 2002:84-A:1174-82.

10. Nagels J, Valstar ER, Stokdijk M, Rozing PM. Patterns of loosening of the glenoid component. J Bone Joint Surg [Br] 2002;84-B:83-7.

11. Neer CS 2nd, Watson KC, Stanton FJ. Recent experience in total shoulder replacement. J Bone Joint Surg [Am] 1982;64-A:319-37.

12. Iannotti JP, Gabriel JP, Schneck SL, et al. The normal glenohumeral relationships: an anatomical study of one hundred and forty shoulders. J Bone Joint Surg [Am] 1992;74-A:491-500.

13. Boileau P, Walch G. The three-dimensional geometry of the proximal humerus: implications for surgical technique and prosthetic design. J Bone Joint Surg $[\mathrm{Br}]$ 1997:79-B:857-65.

14. Nuttall D, Haines JF, Trail II. A study of the micromovement of pegged and keeled glenoid components compared using radiostereometric analysis. J Shoulder Elbow Surg 2007;16(Suppl):65-70.

15. Trail IA, Nuttall D, Stanley JK. Survivorship and radiological analysis of the standard Souter-Strathclyde total elbow arthroplasty. J Bone Joint Surg [Br] 1999;81B:80-4.

16. Walch G, Boulahia A, Boileau P, Kempf JE. Primary glenohumeral osteoarthritis: clinical and radiographic classification: the Aequalis Group. Acta Orthop Belg 1998;64(Suppl 2):46-52.

17. Constant CR, Murley AH. A clinical method of functional assessment of the shoulder. Clin Orthop 1987;214:160-4.

18. Richards R, An K-N, Bigliani LU, et al. A standardised method for the assessment of shoulder function. J Shoulder Elbow Surg 1994;3:347-52.

19. Derbyshire B, Prescott RJ, Porter ML. Notes on the use and interpretation of radiostereometric analysis. Acta Orthop 2009;80:124-30.

20. Valstar ER, Gill R, Ryd L, et al. Guidelines for standardization of radiostereometry (RSA) of implants. Acta Orthop 2005;76:563-72.

21. Sperling JW, Cofield RH, O'Driscoll SW, Torchia ME, Rowland CM. Radiographic assessment of ingrowth total shoulder arthroplasty. J Shoulder Elbow Surg 2000;9:507-13.

22. Rahme H, Mattsson P, Larsson S. Stability of cemented all-polyethylene keeled glenoid components: a radiostereometric study with a two-year follow-up. J Bone Joint Surg [Br] 2004;86-B:856-60.

23. Mileti J, Boardman ND 3rd, Sperling JW, et al. Radiographic analysis of polyethylene glenoid components using modern cementing techniques. J Shoulder Elbow Surg 2004;13:492-8.

24. D'Arco P, Sitler M, Kelly J, et al. Clinical, functional, and radiographic assessments of the conventional and modified Boyd-Anderson surgical procedures for repair of distal biceps tendon ruptures. Am J Sports Med 1998;26:254-61.

25. Wirth MA, Seltzer DG, Rockwood CA Jr. Recurrent posterior glenohumeral dislocation associated with increased retroversion of the glenoid: a case report. Clin Orthop 1994;308:98-101.

26. MacDermid JC, Fess EE, Bell-Krotoski J, et al. A research agenda for hand therapy. J Hand Ther 2002;15:3-15.

27. Matsen FA 3rd, Clinton J, Lynch J, Bertelsen A, Richardson ML. Glenoid component failure in total shoulder arthroplasty. J Bone Joint Surg [Am]2008;90-A:885-96.

28. Gartsman GM, Elkousy HA, Warnock KM, Edwards TB, O'Connor DP. Radiographic comparison of pegged and keeled glenoid components. J Shoulder Elbow Surg 2005;14:252-7.

29. Nyffeler RW, Jost B, Pfirrmann CW, Gerber C. Measurement of glenoid version: conventional radiographs versus computed tomography scans. J Shoulder Elbow Surg 2003;12:493-6.

30. Scalise JJ, Codsi MJ, Bryan J, lannotti JP. The three-dimensional glenoid vault model can estimate normal glenoid version in osteoarthritis. J Shoulder Elbow Surg 2008;17:487-91. 